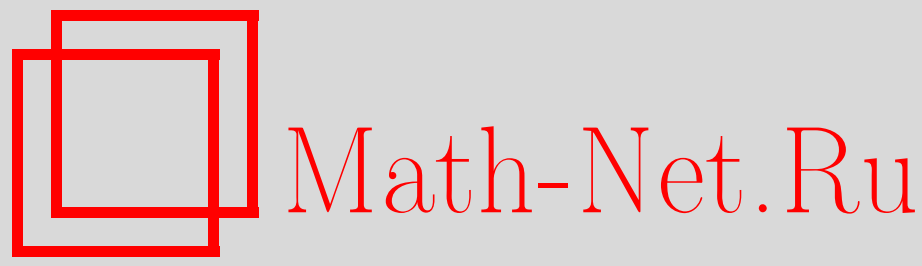

T. В. Скрыпник, Дуальная $R$-матричная интегрируемость, ТМФ, 2008, том 155, номер 1, 147-160

DOI: https://doi.org/10.4213/tmf6200

Использование Общероссийского математического портала Math-Net.Ru подразумевает, что вы прочитали и согласны с пользовательским соглашением http://www . mathnet.ru/rus/agreement

Параметры загрузки:

IP: 54.172 .240 .79

26 апреля 2023 г., 12:36:00

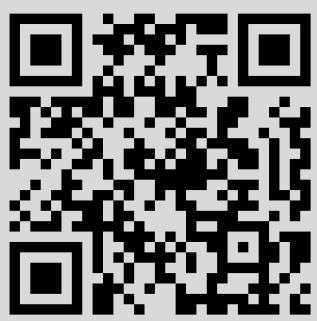




\section{ДУАЛЬНАЯ $R$-МАТРИЧНАЯ ИНТЕГРИРУЕМОСТЬ}

C помощью $R$-оператора на алгебре Ли $\mathfrak{g}$, удовлетворяющего модифицированному классическому уравнению Янга-Бакстера, найдено два множества функций, взаимно коммутирующих относительно исходной скобки Пуассона-Ли на $\mathfrak{g}^{*}$. Детально рассматриваются примеры алгебр Ли $\mathfrak{g}$ с разложением Костанта-Адлера-Симса и треугольным разложением, их $R$-операторы и соответствующие два набора взаимно коммутирующих функций. Найден ответ на вопрос, для каких $R$-операторов построенные наборы функций коммутируют и относительно $R$-скобки. Вкратце обсуждаются интегрируемые уравнения типа Эйлера-Арнольда, для которых построенные коммутирующие функции образуют алгебру первых интегралов движения.

Ключевые слова: алгебры Ли, классические $R$-матрицы, классические интегрируемые системы.

\section{1. ВВЕДЕНИЕ}

Интегрируемость многих уравнений типа Эйлера-Арнольда на дуальном пространстве $\mathfrak{g}^{*}$ алгебры Ли $\mathfrak{g}$ связана с так называемым $R$-оператором или с классической $R$-матрицей. Классическая $R$-матрица представляет собой линейное отображение $R: \mathfrak{g} \rightarrow \mathfrak{g}$, удовлетворяющее модифицированному классическому уравнению Янга-Бакстера [1]

$$
R([R(X), Y]+[X, R(Y)])-[R(X), R(Y)]=[X, Y] \quad \forall X, Y \in \mathfrak{g} .
$$

С помощью $R$-оператора можно определить вторую скобку Ли ( $R$-скобку) на $\mathfrak{g}$, заданную следующей формулой [1]:

$$
[X, Y]_{R} \equiv[R(X), Y]+[X, R(Y)]
$$

где $X, Y \in \mathfrak{g}$, a $[\cdot, \cdot]$ - исходная скобка Ли на $\mathfrak{g}$.

Важность $R$-скобки следует из того, что кольцо $I^{G}\left(\mathfrak{g}^{*}\right) \mathrm{ad}^{*}$-инвариантных функций на $\mathfrak{g}$ представляет собой коммутативную подалгебру относительно второй пуассоновой скобки $\{\cdot, \cdot\}_{R}$ (скобки Пуассона-Ли на $\mathfrak{g}^{*}$, отвечающей лиевой скобке $[\cdot, \cdot]_{R}$ на g) [1]. Это, в свою очередь, позволяет определить гамильтоновы системы на

${ }^{*}$ Институт теоретической физики им. Н. Н. Боголюбова, Киев, Украина. E-mail: tskrypnyk@imath.kiev.ua 
$\left(\mathfrak{g}^{*},\{\cdot, \cdot\}_{R}\right)$, которые изначально допускают независимые взаимно коммутирующие интегралы движения, и эти интегралы оказываются генераторами кольца $I^{G}\left(\mathfrak{g}^{*}\right)$, порождающими нетривиальные гамильтоновы потоки относительно скобки $\{\cdot, \cdot\}_{R}$. Это хорошо известная теория [1], [2], которую мы будем называть прямой $R$-матричной интегрируемостью.

В настоящей работе предлагается другой способ построения алгебр взаимно коммутирующих первых интегралов движения для некоторых уравнений типа ЭйлераАрнольда с использованием понятия классического $R$-оператора. Мы называем этот подход дуальной $R$-матричной интегрируемостью.

Оказывается, что если задан классический $R$-оператор, то можно определить другую алгебру взаимно коммутирующих функций. В противоположность стандартному подходу работы [1], они коммутируют не относительно $R$-скобки $\{\cdot, \cdot\}_{R}$, а относительно исходной скобки Пуассона-Ли $\{\cdot, \cdot\}$. Эти функции также строятся с помощью кольца $I^{G}\left(\mathfrak{g}^{*}\right)$. Более точно, ниже мы доказываем следующую важную теорему (см. теорему 1 ) о том, что функции $I_{k}^{R_{ \pm}}(L) \equiv I_{k}\left(\left(R^{*} \pm 1\right)(L)\right)$, где $I_{k}(L), I_{l}(L) \in I^{G}\left(\mathfrak{g}^{*}\right)$, образуют абелеву подалгебру в $C^{\infty}\left(\mathfrak{g}^{*}\right)$ относительно стандартных скобок Пуассона-Ли $\{\cdot, \cdot\}$ на $\mathfrak{g}^{*}$.

Другими словами, мы строим два набора $\left\{I_{k}^{R_{+}}(L)\right\}$ и $\left\{I_{k}^{R_{-}}(L)\right\}$ функций, коммутирующих относительно скобок $\{\cdot, \cdot\}$, и эти наборы функций также коммутируют друг с другом. Число построенных взаимно коммутирующих функций, таким образом, вдвое больше, чем число независимых генераторов алгебры $I^{G}\left(\mathfrak{g}^{*}\right)$.

В качестве примеров нашей конструкции мы рассмотрим реальные $R$-операторы. Первый пример связан с алгебрами Ли g с так называемым разложением КостантаАдлера-Симса (KАC) в сумму двух подалгебр: $\mathfrak{g}=\mathfrak{g}_{+}+\mathfrak{g}_{-}$, а для соответствующего $R$-оператора имеем $R=P_{+}-P_{-}$(здесь $P_{ \pm}-$операторы проекций на подалгебры $\left.\mathfrak{g}_{ \pm}\right)[1]$. В этом случае наши коммутирующие функции $I_{k}^{R_{ \pm}}(L)$ совпадают с ограничениями коприсоединенных инвариантов $\left\{I_{k}(L)\right\}$ на пространства, дуальные соответствующим алгебрам Ли $\mathfrak{g}_{ \pm}$. Коммутативность таких ограниченных коприсоединенных инвариантов была впервые отмечена в работе [3] (см. подробное доказательство в работе [4]). Таким образом, в частном случае мы обнаруживаем известное утверждение, которое доказало свою пользу в теории солитонных уравнений [3], [4].

Другой интересный пример связан с алгебрами Ли с треуголъным разложением: $\mathfrak{g}=\mathfrak{g}_{+}+\mathfrak{g}_{0}+\mathfrak{g}_{-}$, где $\mathfrak{g}_{ \pm}, \mathfrak{g}_{0}-$ подалгебры и $\mathfrak{g}_{0}$-модули. Покажем, что в этом случае можно определить $R$-оператор с помощью следующей формулы: $R=P_{+}+R_{0}-P_{-}$, где $R_{0}$ - произвольное решение модифицированного уравнения Янга-Бакстера на $\mathfrak{g}_{0}$. (Следует отметить, что в специальном случае петлевой алгебры $s l(2)$, обладающей естественным треугольным разложением, такой $R$-оператор был впервые рассмотрен в работе [5].) В частности, если алгебра $\mathfrak{g}_{0}$ абелева, то в качестве $R_{0}$ можно взять произвольный линейный оператор. В этом случае можно также в явном виде представить построенные наборы коммутирующих функций $I_{k}^{R_{ \pm}}(L)$. Эти функции имеют простой вид и также весьма полезны в теории интегрируемых систем [6].

В настоящей статье мы отвечаем на естественный вопрос, когда построенные коммутативные алгебры коммутируют также относительно $R$-скобки. Мы покажем, что они коммутируют относительно $R$-скобки $\{\cdot, \cdot\}_{R}$ на $\mathfrak{g}^{*}$, если $R$-оператор имеет тип 
$\mathrm{KAC}$ или если он получается с помощью треугольного разложения с абелевой составляющей $\mathfrak{g}_{0}$. Заметим, что коммутативность этих функций относительно $R$-скобки в случае $R$-операторов КАС оказывается гораздо более простым фактом, чем их коммутативность относительно исходной скобки $\{\cdot, \cdot\}$.

Мы обсудим также вкратце приложение наших коммутативных алгебр в теории интегрируемых гамильтоновых систем. В частности, покажем, что соответствующие гамильтоновы уравнения можно переписать в форме Эйлера-Арнольда (в обобщенной лаксовой форме).

Структура работы следующая. В разделе 2 доказывается основная теорема и рассматриваются ее применения в алгебрах Ли с разложением КАС и с треугольным разложением. В разделе 3 исследуется вопрос о коммутативности построенных алгебр относительно $R$-скобок, а возможные приложения полученных результатов в теории интегрируемых систем обсуждаются в разделе 4.

\section{2. КОММУТАТИВНЫЕ ПОДАЛГЕБРЫ И КЛАССИЧЕСКИЙ $R$-ОПЕРАТОР}

2.1. Общий случай. Пусть $\mathfrak{g}$ - алгебра Ли (конечно- или бесконечномерная) со скобкой Ли $[\cdot, \cdot], R: \mathfrak{g} \rightarrow \mathfrak{g}-$ некоторый линейный оператор. Оператор $R$ называется классическим $R$-оператором, если он удовлетворяет модифицированному уравнению Янга-Бакстера (1). Известно [7], [8], [1], что это уравнение можно записать в трех эквивалентных формах:

$$
\begin{aligned}
(R+1)([R(X), Y]+[X, R(Y)]) & =[(R+1)(X),(R+1)(Y)], \\
(R-1)([R(X), Y]+[X, R(Y)]) & =[(R-1)(X),(R-1)(Y)], \\
(R-1)([(R+1)(X),(R+1)(Y)]) & =(R+1)([(R-1)(X),(R-1)(Y)]) .
\end{aligned}
$$

Все три формы записи будут использованы в доказательстве основной теоремы. Также используется обозначение $R_{ \pm} \equiv R \pm 1$.

ЗАмЕчаниЕ 1 . Как следует из равенств (3а), (3б), отображения $R_{ \pm}$задают подалгебры Ли $\mathfrak{g}_{R_{ \pm}} \subset \mathfrak{g}: \mathfrak{g}_{R_{ \pm}}=\operatorname{Im} R_{ \pm}$[7], [8], [1]. Из их определения нетрудно увидеть, что $\mathfrak{g}_{R_{+}}+\mathfrak{g}_{R_{-}}=\mathfrak{g}$, но эта сумма не является в общем случае прямой суммой векторных пространств, т.е. $\mathfrak{g}_{R_{+}} \cap \mathfrak{g}_{R_{-}} \neq 0$.

Обозначим через $\mathfrak{g}^{*}$ пространство, дуальное $\mathfrak{g}$, и через $\langle\cdot, \cdot\rangle: \mathfrak{g}^{*} \times \mathfrak{g} \rightarrow \mathbb{C}-$ спаривание между $\mathfrak{g}^{*}$ и $\mathfrak{g}$. Пусть $\left\{X_{i}\right\}$ - базис алгебры Ли $\mathfrak{g},\left\{X_{i}^{*}\right\}-$ базис в дуальном пространстве $\mathfrak{g}^{*}:\left\langle X_{j}^{*}, X_{i}\right\rangle=\delta_{i j}, L=\sum_{i} L_{i} X_{i}^{*} \in \mathfrak{g}^{*}-$ элемент $\mathfrak{g}^{*}$ общего вида, $L_{i}-$ координатные функции на $\mathfrak{g}^{*}$.

Рассмотрим стандартную скобку Ли-Пуассона между $F_{1}, F_{2} \in C^{\infty}\left(\mathfrak{g}^{*}\right)$ :

$$
\left\{F_{1}(L), F_{2}(L)\right\}=\left\langle L,\left[\nabla F_{1}, \nabla F_{2}\right]\right\rangle,
$$

где

$$
\nabla F_{k}(L)=\sum_{i} \frac{\partial F_{k}(L)}{\partial L_{i}} X_{i}
$$

- так называемый градиент $F_{k}$ со значениями в алгебре. Здесь и далее суммирования предполагаются по всем базисным элементам $\mathfrak{g}$. 
Пусть $R^{*}$ - оператор, дуальный $R$ и действующий в пространстве $\mathfrak{g}^{*}$ :

$$
\left\langle R^{*}(Y), X\right\rangle \equiv\langle Y, R(X)\rangle \quad \forall Y \in \mathfrak{g}^{*}, \quad X \in \mathfrak{g} .
$$

Пусть $I^{G}\left(\mathfrak{g}^{*}\right)$ - кольцо инвариантов коприсоединенного представления алгебры $\mathfrak{g}$. Пусть $I_{k}(L) \in I^{G}\left(\mathfrak{g}^{*}\right)$, т.е. $\left\{I_{k}(L), L_{j}\right\}=0$ для произвольной координатной функции $L_{j}$ или, эквивалентно,

$$
\left\langle\operatorname{ad}_{\nabla I_{k}(L)}^{*} L, X_{j}\right\rangle=0 .
$$

Рассмотрим функции $I_{k}^{R_{ \pm}}(L)$ на $\mathfrak{g}^{*}$, определенные следующими формулами:

$$
I_{k}^{R_{ \pm}}(L) \equiv I_{k}\left(\left(R^{*} \pm 1\right)(L)\right) \equiv I_{k}\left(R_{ \pm}^{*}(L)\right) .
$$

ЗАмечАниЕ 2. Как нетрудно видеть из определения функций $I_{k}^{R_{ \pm}}(L)$, координаты элементов дуального пространства $R_{ \pm}^{*}(L)$ реализуют подалгебры Ли в $\left(\mathfrak{g}^{*},\{\cdot, \cdot\}\right)$, изоморфные $\mathfrak{g}_{R_{ \pm}}$. Поэтому можно интерпретировать функции $I_{k}^{R_{ \pm}}(L)$ как функции на подпространствах $\mathfrak{g}_{R_{ \pm}}^{*}$. Тем не менее, как следует из замечания 1 , оба набора функций $\left\{I_{k}^{R_{+}}(L)\right\}$ и $\left\{I_{l}^{R_{-}}(L)\right\}$ оказываются функциями на полном дуальном пространстве $\mathfrak{g}^{*}$, а не на каких-то его подпространствах.

Очевидно, что, в отличие от функций $I_{k}(L)$, функции $I_{k}^{R_{ \pm}}(L)$ не являются функциями Казимира алгебры Ли $\mathfrak{g}$ или подалгебр Ли $\mathfrak{g}_{R_{ \pm}}$. Тем не менее выполнена следующая теорема.

Теорема 1. Функиии $\left\{I_{k}^{R_{+}}(L)\right\} u\left\{I_{l}^{R_{-}}(L)\right\}$, где $I_{k}(L), I_{l}(L) \in I^{G}\left(\mathfrak{g}^{*}\right)$, порожсдают абелеву подалгебру в $C^{\infty}\left(\mathfrak{g}^{*}\right)$ относительно стандартных скобок Пуассона-Ли $\{\cdot, \cdot\} н а \mathfrak{g}^{*}:$

1) $\left\{I_{k}^{R_{+}}(L), I_{l}^{R_{+}}(L)\right\}=0$,

2) $\left\{I_{k}^{R_{-}}(L), I_{l}^{R_{-}}(L)\right\}=0$,

3) $\left\{I_{k}^{R_{+}}(L), I_{l}^{R_{-}}(L)\right\}=0$.

Для доказательства этой теоремы необходима следующая вспомогательная леммa.

Лемма. Пусть $A \in \operatorname{End}(\mathfrak{g})$, a $A^{*}$ - ее сопряженный оператор. Пусть любая бункиия $F \in C^{\infty}\left(\mathfrak{g}^{*}\right)$ задает следуюшую функиию на $\mathfrak{g}^{*}:$

$$
F^{A}(L) \equiv F\left(A^{*}(L)\right) .
$$

Тогда выполнено соотношение

$$
\nabla F^{A}(L)=A\left(\left.\nabla F(L)\right|_{L=A^{*}(L)}\right)
$$

ЗАмечАние 3. Смысл обозначения $\left.\nabla F(L)\right|_{L=A^{*}(L)}$ в том, что ограничение элемента $L$ на подпространство $\operatorname{Im} A^{*}$ осуществляется после вычисления алгеброзначного градиента $F$ (операции взятия алгеброзначного градиента и ограничения функции на подпространство, вообще говоря, не коммутативны). 
ДокАЗАТЕЛЬСтво ЛЕммы. Заметим, что справедливы равенства $A\left(X_{i}\right)=$ $\sum_{j} A_{i}^{j} X_{j}, A^{*}\left(X_{i}^{*}\right)=\sum_{j}\left(A^{*}\right)_{i}^{j} X_{j}^{*}$ и, как легко вывести из определения дуального оператора, $\left(A^{*}\right)_{i}^{j}=A_{j}^{i}$. Поэтому получим

$$
A^{*}(L)=\sum_{i} L_{i} A^{*}\left(X_{i}^{*}\right)=\sum_{i, j} L_{i}\left(A^{*}\right)_{i}^{j} X_{j}^{*} \sum_{i, j} L_{i} A_{j}^{i} X_{j}^{*} \equiv \sum_{j} A\left(L_{j}\right) X_{j}^{*},
$$

где $A\left(L_{j}\right) \equiv \sum_{i} A_{j}^{i} L_{i}$.

Рассмотрим функции $F(L)$ и $F^{A}(L)$ и их матричные градиенты более подробно. Имеем $F(L)=F\left(L_{1}, L_{2}, \ldots\right), F^{A}(L)=F\left(A\left(L_{1}\right), A\left(L_{2}\right), \ldots\right)$. Тем самым получим

$$
\begin{aligned}
\nabla F^{A}(L) & =\sum_{i} \frac{\partial F\left(A\left(L_{1}\right), A\left(L_{2}\right), \ldots\right)}{\partial L_{i}} X_{i}=\sum_{i, j} \frac{\partial F\left(A\left(L_{1}\right), A\left(L_{2}\right), \ldots\right)}{\partial A\left(L_{j}\right)} \frac{\partial A\left(L_{j}\right)}{\partial L_{i}} X_{i}= \\
& =\sum_{i, j} \frac{\partial F\left(A\left(L_{1}\right), A\left(L_{2}\right), \ldots\right)}{\partial A\left(L_{j}\right)} A_{j}^{i} X_{i}=\sum_{j} \frac{\partial F\left(A\left(L_{1}\right), A\left(L_{2}\right), \ldots\right)}{\partial A\left(L_{j}\right)} A\left(X_{j}\right)= \\
& =A\left(\left.\nabla F(L)\right|_{L=A^{*}(L)}\right) .
\end{aligned}
$$

Лемма доказана.

ДокАЗАтЕЛЬСтво теоремы 1. Докажем утверждение 1, т.е. покажем, что $\left\{I_{k}^{R_{+}}(L), I_{l}^{R_{+}}(L)\right\}=0$, если $I_{k}(L), I_{l}(L) \in I^{G}(\mathfrak{g})$. Применяя предыдущую лемму, получим

$$
\begin{aligned}
\left\{I_{k}^{R_{+}}(L), I_{l}^{R_{+}}(L)\right\} & =\left\langle L,\left[\nabla I_{k}^{R_{+}}(L), \nabla I_{l}^{R_{+}}(L)\right]\right\rangle= \\
& =\left\langle L,\left[R_{+}\left(\left.\nabla I_{k}(L)\right|_{L=R_{+}^{*}(L)}\right), R_{+}\left(\left.\nabla I_{l}(L)\right|_{L=R_{+}^{*}(L)}\right)\right]\right\rangle .
\end{aligned}
$$

C помощью равенства (3a) последнее выражение можно переписать в следующем виде:

$$
\begin{aligned}
\left\langle L, R_{+}(\right. & {\left[R\left(\left.\nabla I_{k}(L)\right|_{L=R_{+}^{*}(L)}\right),\left.\nabla I_{l}(L)\right|_{L=R_{+}^{*}(L)}\right]+} \\
& \left.\left.+\left[\left.\nabla I_{k}(L)\right|_{L=R_{+}^{*}(L)}, R\left(\left.\nabla I_{l}(L)\right|_{L=R_{+}^{*}(L)}\right)\right]\right)\right\rangle= \\
=\langle & R_{+}^{*} L,\left[R\left(\left.\nabla I_{k}(L)\right|_{L=R_{+}^{*}(L)}\right),\left.\nabla I_{l}(L)\right|_{L=R_{+}^{*}(L)}\right]+ \\
& \left.+\left[\left.\nabla I_{k}(L)\right|_{L=R_{+}^{*}(L)}, R\left(\left.\nabla I_{l}(L)\right|_{L=R_{+}^{*} L}\right)\right]\right\rangle= \\
=- & \left\langle\operatorname{ad}_{\left.\nabla I_{l}(L)\right|_{L=R_{+}^{*}(L)}} R_{+}^{*}(L), R\left(\left.\nabla I_{k}(L)\right|_{L=R_{+}^{*}(L)}\right)\right\rangle+ \\
& +\left\langle\operatorname{ad}_{\left.\nabla I_{k}(L)\right|_{L=R_{+}^{*}(L)}} R_{+}^{*}(L), R\left(\left.\nabla I_{l}(L)\right|_{L=R_{+}^{*}(L)}\right)\right\rangle .
\end{aligned}
$$

С другой стороны,

$\left\langle\operatorname{ad}_{\left.\nabla I_{l}(L)\right|_{L=R_{+}^{*}(L)} ^{*}} R_{+}^{*}(L), R\left(\left.\nabla I_{k}(L)\right|_{L=R_{+}^{*}(L)}\right)\right\rangle=\left.\left\langle\operatorname{ad}_{\nabla I_{l}(L)}^{*} L, R\left(\nabla I_{k}(L)\right)\right\rangle\right|_{L=R_{+}^{*}(L)}=0$,

поскольку $I_{l}(L)$ является инвариантной функцией. Поэтому первое слагаемое в $(8)$ равно нулю. По той же причине второе слагаемое в (8) также равно нулю. Следовательно, $\left\{I_{k}^{R_{+}}(L), I_{l}^{R_{+}}(L)\right\}=0$. Утверждение 1 доказано. 
Утверждение 2 доказывается аналогично с помощью равенства (3б) вместо равенства (3a).

Докажем утверждение 3, т.е. покажем, что

$$
\left\{I_{k}^{R_{+}}(L), I_{l}^{R_{-}}(L)\right\} \equiv\left\langle L,\left[\nabla I_{k}^{R_{+}}(L), \nabla I_{l}^{R_{-}}(L)\right]\right\rangle=0 .
$$

Для этого воспользуемся равенством id $=\left(R_{+}-R_{-}\right) / 2$, а потому $2 L=$ $\left(R_{+}^{*}-R_{-}^{*}\right)(L)$, и перепишем двойное выражение для $\left\{I_{k}^{R_{+}}(L), I_{l}^{R_{-}}(L)\right\}$ в следующем виде:

$$
\begin{aligned}
\left\langle R_{+}^{*}\right. & \left.(L),\left[\nabla I_{k}^{R_{+}}(L), \nabla I_{l}^{R_{-}}(L)\right]\right\rangle-\left\langle R_{-}^{*}(L),\left[\nabla I_{k}^{R_{+}}(L), \nabla I_{l}^{R_{-}}(L)\right]\right\rangle= \\
& =\left\langle R_{+}^{*}(L),\left[R_{+}\left(\left.\nabla I_{k}(L)\right|_{L=R_{+}^{*}(L)}\right), R_{-}\left(\left.\nabla I_{l}(L)\right|_{L=R_{-}^{*}(L)}\right)\right]\right\rangle- \\
& -\left\langle R_{-}^{*}(L),\left[R_{+}\left(\left.\nabla I_{k}(L)\right|_{L=R_{+}^{*}(L)}\right), R_{-}\left(\left.\nabla I_{l}(L)\right|_{L=R_{-}^{*}(L)}\right)\right]\right\rangle .
\end{aligned}
$$

Рассмотрим по отдельности два слагаемых в правой части этого тождества. Имеem

$$
\begin{aligned}
& \left\langle R_{+}^{*}(L),\left[R_{+}\left(\left.\nabla I_{k}(L)\right|_{L=R_{+}^{*}(L)}\right), R_{-}\left(\left.\nabla I_{l}(L)\right|_{L=R_{-}^{*}(L)}\right)\right]\right\rangle= \\
& \quad=2\left\langle R_{+}^{*}(L),\left[\left.\nabla I_{k}(L)\right|_{L=R_{+}^{*}(L)}, R_{-}\left(\left.\nabla I_{l}(L)\right|_{L=R_{-}^{*}(L)}\right)\right]\right\rangle+ \\
& \quad+\left\langle R_{+}^{*}(L),\left[R_{-}\left(\left.\nabla I_{k}(L)\right|_{L=R_{+}^{*}(L)}\right), R_{-}\left(\left.\nabla I_{l}(L)\right|_{L=R_{-}^{*}(L)}\right)\right]\right\rangle,
\end{aligned}
$$

где было использовано то, что $R_{+}\left(\nabla I_{k}(L)\right)=2 \nabla I_{k}(L)+R_{-}\left(\nabla I_{k}(L)\right)$. Первое слагаемое в правой части (9) можно переписать в виде

$$
\begin{aligned}
& 2\left\langle R_{+}^{*}(L),\left[\left.\nabla I_{k}(L)\right|_{L=R_{+}^{*}(L)}, R_{-}\left(\left.\nabla I_{l}(L)\right|_{L=R_{-}^{*}(L)}\right)\right]\right\rangle= \\
& \quad=2\left\langle\operatorname{ad}_{\left.\nabla I_{k}(L)\right|_{L=R_{+}^{*}(L)} ^{*}} R_{+}^{*}(L), R_{-}\left(\left.\nabla I_{l}(L)\right|_{L=R_{-}^{*}(L)}\right)\right\rangle= \\
& \quad=2\left\langle\left.\left(\operatorname{ad}_{\nabla I_{k}(L)}^{*} L\right)\right|_{L=R_{+}^{*}(L)}, X\right\rangle,
\end{aligned}
$$

где $X \equiv R_{-}\left(\left.\nabla I_{l}(L)\right|_{L=R_{-}^{*}(L)}\right)$. Принимая в расчет инвариантность функции $I_{k}(L)$, убеждаемся, что это слагаемое равно нулю. Следовательно,

$$
\begin{aligned}
\left\langle R_{+}^{*}\right. & \left.(L),\left[R_{+}\left(\left.\nabla I_{k}(L)\right|_{L=R_{+}^{*}(L)}\right), R_{-}\left(\left.\nabla I_{l}(L)\right|_{L=R_{-}^{*}(L)}\right)\right]\right\rangle= \\
\quad & \left\langle R_{+}^{*}(L),\left[R_{-}\left(\left.\nabla I_{k}(L)\right|_{L=R_{+}^{*}(L)}\right), R_{-}\left(\left.\nabla I_{l}(L)\right|_{L=R_{-}^{*}(L)}\right)\right]\right\rangle= \\
& =\left\langle L, R_{+}\left(\left[R_{-}\left(\left.\nabla I_{k}(L)\right|_{L=R_{+}^{*}(L)}\right), R_{-}\left(\left.\nabla I_{l}(L)\right|_{L=R_{-}^{*}(L)}\right)\right]\right)\right\rangle .
\end{aligned}
$$

Аналогично легко показать, что

$$
\begin{aligned}
& \left\langle R_{-}^{*}(L),\left[R_{+}\left(\left.\nabla I_{k}(L)\right|_{L=R_{+}^{*}(L)}\right), R_{-}\left(\left.\nabla I_{l}(L)\right|_{L=R_{-}^{*}(L)}\right)\right]\right\rangle= \\
& \quad=\left\langle L, R_{-}\left(\left[R_{+}\left(\left.\nabla I_{k}(L)\right|_{L=R_{+}^{*}(L)}\right), R_{+}\left(\left.\nabla I_{l}(L)\right|_{L=R_{-}^{*}(L)}\right)\right]\right)\right\rangle .
\end{aligned}
$$


Получим при этом, что

$$
\left\{I_{k}^{R_{+}}(L), I_{l}^{R_{-}}(L)\right\}=\left\langle L, R_{+}\left(\left[R_{-}(X), R_{-}(Y)\right]\right)-R_{-}\left(\left[R_{+}(X), R_{+}(Y)\right]\right)\right\rangle=0,
$$

где $\left.X \equiv \nabla I_{k}(L)\right|_{L=R_{+}^{*}(L)},\left.Y \equiv \nabla I_{l}(L)\right|_{L=R_{-}^{*}(L)}$ и было использовано равенство (3в). Это доказывает утверждение 3 .

Теорема доказана.

Эта теорема предоставляет нам широкую абелеву подалгебру в пространстве $\left(C^{\infty}\left(\mathfrak{g}^{*}\right),\{\cdot, \cdot\}\right)$. В следующих разделах рассматриваются несколько примеров $R$-операторов и соответствующих абелевых подалгебр.

2.2. Случай алгебр Ли с разложением КАС. Рассмотрим случай алгебр Ли $\mathfrak{g}$ с разложением КАС в прямую сумму двух векторных подпространств $\mathfrak{g}_{ \pm}$: $\mathfrak{g}=\mathfrak{g}_{+}+\mathfrak{g}_{-}$, где подпространства $\mathfrak{g}_{ \pm}$суть замкнутые подалгебры Ли. Пусть $P_{ \pm}-$ проекционные операторы на соответствующие подалгебры $\mathfrak{g}_{ \pm}$. Тогда известно [1], что в этом случае можно определить так называемую $R$-матрицу $\mathrm{KAC}$ :

$$
R=P_{+}-P_{-}
$$

Легко видеть, что в этом случае $R_{+}=1+R=2 P_{+}, R_{-}=R-1=-2 P_{-}$пропорциональны проекционным операторам на подалгебры $\mathfrak{g}_{ \pm}$. Получим, что $\mathfrak{g}_{R_{ \pm}} \equiv \mathfrak{g}_{ \pm}$ и $\mathfrak{g}_{R_{+}} \cap \mathfrak{g}_{R_{-}}=0$.

Предположим, что генераторы кольца инвариантных функций $I_{k}(L)$ можно выбрать в виде однородных многочленов. Функции $I_{k}^{R_{ \pm}}(L)$ тогда принимают следующий простой вид:

$$
I_{k}^{R_{ \pm}}(L) \equiv I_{k}^{ \pm}(L) \equiv I_{k}\left(L_{ \pm}\right), \quad L_{ \pm} \equiv P_{ \pm}^{*} L
$$

т.е. $I_{k}^{ \pm}(L)$ представляют собой ограничения коприсоединенных инвариантов на дуальные пространства подалгебры Ли $\mathfrak{g}_{ \pm}$. Тем самым имеет место следующее следствие теоремы 1.

СлЕДСТВИЕ 1. Функиии $\left\{I_{k}\left(L_{+}\right)\right\} u\left\{I_{l}\left(L_{-}\right)\right\}$, где $I_{k}(L), I_{l}(L) \in I^{G}\left(\mathfrak{g}^{*}\right)$, образуют абелеву подалгебру в $C^{\infty}\left(\mathfrak{g}^{*}\right)$ относительно скобки Пуассона-Ли на $\mathfrak{g}^{*}$ :

1) $\left\{I_{k}\left(L_{+}\right), I_{l}\left(L_{+}\right)\right\}=0$,

2) $\left\{I_{k}\left(L_{-}\right), I_{l}\left(L_{-}\right)\right\}=0$,

3) $\left\{I_{k}\left(L_{+}\right), I_{l}\left(L_{-}\right)\right\}=0$.

ЗАмЕчАниЕ 4. Это следствие задает чрезвычайно простой способ построения набора коммутирующих интегралов на $\mathfrak{g}=\mathfrak{g}_{+}+\mathfrak{g}_{-}$, который просто совпадает с ограничением коприсоединенных инвариантов на дуальное пространство каждой подалгебры в разложении. Заметим, что утверждения 1, 2 следствия 1 можно также получить с помощью стандартного $R$-матричного подхода [1]. Утверждение 3 не следует из стандартной схемы КАС. Его справедливость была впервые сформулирована без доказательства в работе [3]. Подробное доказательство утверждения 3 в этом случае было дано в работе [4]. 
2.3. Случай алгебр Ли с треугольным разложением. Рассмотрим случай алгебр Ли $\mathfrak{g}$ с так называемым треуголъным разложением

$$
\mathfrak{g}=\mathfrak{g}_{+}+\mathfrak{g}_{0}+\mathfrak{g}_{-},
$$

где сумма представляет собой прямую сумму векторных пространств, $\mathfrak{g}_{ \pm}$и $\mathfrak{g}_{0}-$ замкнутые подалгебры и $\mathfrak{g}_{ \pm}-\mathfrak{g}_{0}$-модули. Разумеется, в этом случае разложения $\mathrm{KAC}$ (два из них) алгебры Ли $\mathfrak{g}$ и $R$-операторы КАC также существуют, однако треугольное разложение (10) обеспечивает более широкие возможности.

Верно следующее предложение.

ПреДЛОЖЕНИЕ 1. Пусть $R_{0}$ - решение модифицированного уравнения ЯнгаБакстера (1) на $\mathfrak{g}_{0}$. Тогда тензор

$$
R=P_{+}+R_{0}-P_{-},
$$

где $P_{ \pm}$- проекиионные операторы на подалгебры $\mathfrak{g}_{ \pm}$, есть решение модибицированного уравнения Янга-Бакстера (1) на $\mathfrak{g}$.

ДокАЗАтЕльство. Пусть $X=X_{+}+X_{0}+X_{-}, Y=Y_{+}+Y_{0}+Y_{-}-$разложение $X, Y \in \mathfrak{g}$ такое, что $X_{ \pm} \in \mathfrak{g}_{ \pm}, X_{0} \in \mathfrak{g}_{0}$. Тогда $R(X)=X_{+}+R_{0}\left(X_{0}\right)-X_{-}, R(Y)=$ $Y_{+}+R_{0}\left(Y_{0}\right)-Y_{-}$.

Рассмотрим выражение

$$
R([R(X), Y]+[X, R(Y)])-[R(X), R(Y)]-[X, Y] .
$$

С помощью прямых вычислений получим

$$
\begin{aligned}
{[R(X), Y]+[X, R(Y)]=2 } & \left(\left[X_{+}, Y_{+}\right]-\left[X_{-}, Y_{-}\right]\right)+\left[\left(R_{0}+1\right)\left(X_{0}\right), Y_{+}\right]+ \\
& +\left[X_{+},\left(R_{0}+1\right)\left(Y_{0}\right)\right]+\left[\left(R_{0}-1\right)\left(X_{0}\right), Y_{-}\right]+ \\
& +\left[X_{-},\left(R_{0}-1\right) Y_{0}\right]+\left[X_{0}, R_{0}\left(Y_{0}\right)\right]+\left[R_{0}\left(X_{0}\right), Y_{0}\right] .
\end{aligned}
$$

Учитывая, что $\mathfrak{g}_{ \pm}$и $\mathfrak{g}_{0}-$ подалгебры и $\mathfrak{g}_{ \pm}-\mathfrak{g}_{0}$-модули, получим

$$
\begin{aligned}
& R([R(X), Y]+[X, R(Y)])=2\left(\left[X_{+}, Y_{+}\right]+\left[X_{-}, Y_{-}\right]\right)+\left[\left(R_{0}+1\right)\left(X_{0}\right), Y_{+}\right]+ \\
& \quad+\left[X_{+},\left(R_{0}+1\right)\left(Y_{0}\right)\right]-\left[\left(R_{0}-1\right)\left(X_{0}\right), Y_{-}\right]-\left[X_{-},\left(R_{0}-1\right) Y_{0}\right]+ \\
& \quad+R_{0}\left(\left[X_{0}, R_{0}\left(Y_{0}\right)\right]+\left[R_{0}\left(X_{0}\right), Y_{0}\right]\right) .
\end{aligned}
$$

С другой стороны, прямые вычисления дают

$$
\begin{aligned}
& {[R(X), R(Y)]+[X, Y]=2\left(\left[X_{+}, Y_{+}\right]+\left[X_{-}, Y_{-}\right]\right)+\left[\left(1+R_{0}\right)\left(X_{0}\right), Y_{+}\right]+} \\
& \quad+\left[X_{+},\left(1+R_{0}\right) Y_{0}\right]-\left[X_{-},\left(R_{0}-1\right)\left(Y_{0}\right)\right]-\left[\left(R_{0}-1\right)\left(X_{0}\right), Y_{-}\right]+ \\
& \quad+\left[R_{0}\left(X_{0}\right), R_{0}\left(Y_{0}\right)\right]+\left[X_{0}, Y_{0}\right] .
\end{aligned}
$$

Таким образом, получим, что

$$
R([R(X), Y]+[X, R(Y)])-[R(X), R(Y)]-[X, Y]=0
$$

тогда и только тогда, когда $R_{0}$ удовлетворяет уравнению

$$
R_{0}\left(\left[X_{0}, R_{0}\left(Y_{0}\right)\right]+\left[R_{0}\left(X_{0}\right), Y_{0}\right]\right)-\left[R_{0}\left(X_{0}\right), R_{0}\left(Y_{0}\right)\right]-\left[X_{0}, Y_{0}\right]=0 .
$$

Предложение доказано. 
ЗАмечаниЕ 5 . В случае, когда $R_{0}= \pm \mathrm{id}_{0}$ (очевидно, $R_{0}= \pm \mathrm{id}_{0}$ являются решениями уравнения (12)), получим, что $R$-матрица (11) переходит в стандартную $R$-матрицу $\mathrm{KAC,} \mathrm{отвечающую} \mathrm{разложению} \mathrm{KAC}$

$$
\mathfrak{g}=\left(\mathfrak{g}_{+}+\mathfrak{g}_{0}\right)+\mathfrak{g}_{-} \quad \text { или } \quad \mathfrak{g}=\mathfrak{g}_{+}+\left(\mathfrak{g}_{0}+\mathfrak{g}_{-}\right) .
$$

Тем не менее рассматриваемый случай треугольного разложения содержит и другие интересные возможности. Одна из них дается следующим предложением.

СЛЕДСтвиЕ 2. Пусть подалгебра Ли $\mathfrak{g}_{0}$ абелева. Тогда комбиначия

$$
R=P_{+}+\rho_{0}-P_{-}
$$

дает решение уравнения (1) для любого тензора $\rho_{0}$ на $\mathfrak{g}_{0}$.

ДокАзАтЕльство. Доказательство следует из того, что в случае абелевых алгебр Ли $\mathfrak{g}_{0}$ любой тензор $R_{0}=\rho_{0}$ оказывается решением модифицированного уравнения Янга-Бакстера (1) на $\mathfrak{g}_{0}$.

Другая интересная возможность возникает, когда $\mathfrak{g}_{0}-$ конечномерная полупростая алгебра Ли. Были найдены разнообразные решения модифицированного классического уравнения Янга-Бакстера на конечномерных полупростых алгебрах Ли [7], [1], и эти решения можно применить для построения (с помощью формулы (11)) новых неантисимметричных решений модифицированных классических уравнений Янга-Бакстера на $\mathfrak{g}$.

Рассмотрим более детально построенные алгебры интегралов движения, отвечающих рассмотренным классическим $R$-матрицам. В случае $R$-матрицы (11) имеем

$$
R_{+}=2 P_{+}+\left(P_{0}+R_{0}\right), \quad R_{-}=-\left(2 P_{-}+\left(P_{0}-R_{0}\right)\right) .
$$

Таким образом, в этом случае $\mathfrak{g}_{R_{ \pm}}=\mathfrak{g}_{ \pm}+\operatorname{Im}\left(R_{0}\right)_{ \pm}$, где $\left(R_{0}\right)_{ \pm}=\left(R_{0} \pm P_{0}\right)-$ $R_{ \pm}$-операторы на $\mathfrak{g}_{0}$ и $\mathfrak{g}_{R_{+}} \cap \mathfrak{g}_{R_{-}}=\operatorname{Im}\left(R_{0}\right)_{+} \cap \operatorname{Im}\left(R_{0}\right)_{-}$.

В случае однородных инвариантов $I_{k}(L)$ функции $I_{k}^{R_{ \pm}}(L)$ принимают вид

$$
I_{k}^{R_{ \pm}}(L) \equiv I_{k}^{R_{0, \pm}}(L)=I_{k}\left(L_{ \pm}+\frac{1 \pm R_{0}^{*}}{2}\left(L_{0}\right)\right), \quad L_{ \pm} \equiv P_{ \pm}^{*} L, \quad L_{0} \equiv P_{0}^{*} L .
$$

Если известно решение $R_{0}$ на $\mathfrak{g}_{0}$ (например, в случае, когда алгебра $\mathfrak{g}_{0}$ абелева) и известны коприсоединенные инварианты, функции $I_{k}^{R_{ \pm}}(L)$ оказываются вполне конкретно заданными. При этом имеет место следующее следствие теоремы 1.

СлЕДСТвиЕ 3. Функиии $\left\{I_{k}^{R_{0,+}}(L)\right\} u\left\{I_{l}^{R_{0,-}}(L)\right\}$, где $I_{k}(L), I_{l}(L) \in I^{G}\left(\mathfrak{g}^{*}\right)$, образуют абелеву подалгебру в $C^{\infty}\left(\mathfrak{g}^{*}\right)$ относительно скобки Пуассона-Ли на $\mathfrak{g}^{*}$ :

1) $\left\{I_{k}^{R_{0,+}}(L), I_{l}^{R_{0,+}}(L)\right\}=0$,

2) $\left\{I_{k}^{R_{0,-}}(L), I_{l}^{R_{0,-}}(L)\right\}=0$,

3) $\left\{I_{k}^{R_{0,+}}(L), I_{l}^{R_{0,-}}(L)\right\}=0$.

ЗАмечАниЕ 6 . В случае, когда $R_{0}= \pm \mathrm{id}_{0}$ и функции $I_{k}$ однородны, мы снова приходим к функциям $I_{k}^{ \pm}(L)$, которые могут быть получены при нашем подходе в случае двух разложений КАС алгебры Ли g. В других случаях, полученных в этом разделе, коммутирующие интегралы отличаются от тех, которые были получены с помощью разложения КАС. 


\section{3. КОММУТАТИВНОСТЬ И $R$-СКОБКА}

В данной работе нас в основном интересуют первоначальная скобка Ли $[\cdot, \cdot]$ на $\mathfrak{g}$, соответствующие скобки Пуассона-Ли $\{\cdot, \cdot\}$ на $\mathfrak{g}^{*}$, равно как и функции, коммутирующие относительно такой "первоначальной" скобки Пуассона-Ли. Тем не менее обратимся теперь к естественному вопросу, когда построенные коммутативные алгебры оказываются также коммутирующими относительно $R$-матричной скобки.

Как уже отмечалось, с помощью классической $R$-матрицы, можно задать новую скобку Ли на $\mathfrak{g}[1]$ :

$$
[X, Y]_{R} \equiv[R(X), Y]+[X, R(Y)]
$$

и определить соответствующую структуру Пуассона-Ли $\{\cdot, \cdot\}_{R}$ с помощью формулы

$$
\left\{F_{1}(L), F_{2}(L)\right\}_{R}=\left\langle L,\left[\nabla F_{1}, \nabla F_{2}\right]_{R}\right\rangle,
$$

где

$$
\nabla F_{k}(L)=\sum_{i} \frac{\partial F_{k}(L)}{\partial L_{i}} X_{i}
$$

- стандартный алгеброзначный градиент $F_{k}$.

Хорошо известно [1], что набор функций Казимира $\left\{I_{k}(L)\right\}$ для скобок $\{\cdot, \cdot\}$ образует абелеву подалгебру относительно $R$-скобок $\{\cdot, \cdot\}_{R}$. Ответим теперь на вопрос, когда функции $\left\{I_{k}^{R_{+}}(L)\right\}$ и $\left\{I_{l}^{R_{-}}(L)\right\}$ образуют абелеву подалгебру по отношению не только к скобке $\{\cdot, \cdot\}$, но и к $R$-матричной скобке $\{\cdot, \cdot\}_{R}$.

Справедлива следующая теорема.

Tеорема 2. Функиии $\left\{I_{k}^{R_{+}}(L)\right\} u\left\{I_{l}^{R_{-}}(L)\right\}$, где $I_{k}(L), I_{l}(L) \in I^{G}\left(\mathfrak{g}^{*}\right)$, образуют абелеву подалгебру в $C^{\infty}\left(\mathfrak{g}^{*}\right)$ относительно $R$-скобок $\{\cdot, \cdot\}_{R}$ на $\mathfrak{g}^{*}$, если $R$ имеет mип $\mathrm{KAC}\left(R=P_{+}-P_{-}\right)$или если $R$ получается из треугольного разложения $\left(R=P_{+}+R_{0}-P_{-}\right)$с абелевой подалгеброй $\mathfrak{g}_{0}$.

ДоКАЗАТЕЛЬСтво. Для доказательства теоремы необходимо явно вычислить скобки Пуассона $\left\{I_{k}^{R_{ \pm}}(L), I_{l}^{R_{ \pm}}(L)\right\}_{R}$ и $\left\{I_{k}^{R_{ \pm}}(L), I_{l}^{R_{\mp}}(L)\right\}_{R}$. Имеем

$$
\begin{aligned}
\left\{I_{k}^{R_{ \pm}}(L), I_{l}^{R_{ \pm}}(L)\right\}_{R} & =\left\langle L,\left[\nabla I_{k}^{R_{ \pm}}(L), \nabla I_{l}^{R_{ \pm}}(L)\right]_{R}\right\rangle= \\
& =\left\langle L,\left[R_{ \pm}\left(\left.\nabla I_{k}(L)\right|_{L=R_{ \pm}^{*}(L)}\right), R_{ \pm}\left(\left.\nabla I_{l}(L)\right|_{L=R_{ \pm}^{*}(L)}\right)\right]_{R}\right\rangle .
\end{aligned}
$$

Применяя равенства (3a), (3б) и очевидный факт, что $R$ коммутирует с $R_{ \pm}$, можно легко вывести следующее равенство:

$$
\left[R_{ \pm}(X), R_{ \pm}(Y)\right]_{R}=R_{ \pm}\left(\left[R^{2}(X), Y\right]+2[R(X), R(Y)]+\left[X, R^{2}(Y)\right]\right) .
$$

Используя это равенство и то, что $I_{k}(L)$ и $I_{l}(L)$ представляют собой инвариантные функции, можно упростить выражение для $\left\{I_{k}^{R_{ \pm}}(L), I_{l}^{R_{ \pm}}(L)\right\}_{R}$, приведя его к следующему виду:

$$
\left\{I_{k}^{R_{ \pm}}(L), I_{l}^{R_{ \pm}}(L)\right\}_{R}=2\left\langle R_{ \pm}^{*}(L),\left[R\left(\left.\nabla I_{k}(L)\right|_{L=R_{ \pm}^{*}(L)}\right), R\left(\left.\nabla I_{l}(L)\right|_{L=R_{ \pm}^{*}(L)}\right)\right]\right\rangle .
$$


С учетом модифицированного уравнения Янга-Бакстера для $R$ и того, что $I_{k}(L)$ представляет собой функцию, инвариантную относительно скобок $\{\cdot, \cdot\}$, окончательно получим

$$
\left\{I_{k}^{R_{ \pm}}(L), I_{l}^{R_{ \pm}}(L)\right\}_{R}=2\left\langle R^{*} R_{ \pm}^{*}(L),\left[\left.\nabla I_{k}(L)\right|_{L=R_{ \pm}^{*}(L)},\left.\nabla I_{l}(L)\right|_{L=R_{ \pm}^{*}(L)}\right]_{R}\right\rangle .
$$

Прямые вычисления дают

$$
\begin{aligned}
2\left\{I_{k}^{R_{+}}(L), I_{l}^{R_{-}}(L)\right\}_{R} \equiv\left\langle 2 L,\left[\nabla I_{k}^{R_{+}}(L), \nabla I_{l}^{R_{-}}(L)\right]_{R}\right\rangle= \\
=\left\langle R_{+}^{*}(L),\left[\nabla I_{k}^{R_{+}}(L), \nabla I_{l}^{R_{-}}(L)\right]_{R}\right\rangle-\left\langle R_{-}^{*}(L),\left[\nabla I_{k}^{R_{+}}(L), \nabla I_{l}^{R_{-}}(L)\right]_{R}\right\rangle= \\
=\left\langle R_{+}^{*}(L),\left[R_{+}\left(\left.\nabla I_{k}(L)\right|_{L=R_{+}^{*}(L)}\right), R_{-}\left(\left.\nabla I_{l}(L)\right|_{L=R_{-}^{*}(L)}\right)\right]_{R}\right\rangle- \\
\quad-\left\langle R_{-}^{*}(L),\left[R_{+}\left(\left.\nabla I_{k}(L)\right|_{L=R_{+}^{*}(L)}\right), R_{-}\left(\left.\nabla I_{l}(L)\right|_{L=R_{-}^{*}(L)}\right)\right]_{R}\right\rangle .
\end{aligned}
$$

Рассмотрим по отдельности два слагаемых в правой части этого тождества. Имеем

$$
\begin{aligned}
\left\langle R_{+}^{*}(L),\left[R_{+}\left(\left.\nabla I_{k}(L)\right|_{L=R_{+}^{*}(L)}\right), R_{-}\left(\left.\nabla I_{l}(L)\right|_{L=R_{-}^{*}(L)}\right)\right]_{R}\right\rangle= \\
=2\left\langle R_{+}^{*}(L),\left[\left.\nabla I_{k}(L)\right|_{L=R_{+}^{*}(L)}, R_{-}\left(\left.\nabla I_{l}(L)\right|_{L=R_{-}^{*}(L)}\right)\right]_{R}\right\rangle+ \\
\quad+\left\langle R_{+}^{*}(L),\left[R_{-}\left(\left.\nabla I_{k}(L)\right|_{L=R_{+}^{*}(L)}\right), R_{-}\left(\left.\nabla I_{l}(L)\right|_{L=R_{-}^{*}(L)}\right)\right]_{R}\right\rangle,
\end{aligned}
$$

где было использовано то, что $R_{+}\left(\nabla I_{k}(L)\right)=2 \nabla I_{k}(L)+R_{-}\left(\nabla I_{k}(L)\right)$.

Используя то, что по определению $[X, Y]_{R}=[R(X), Y]+[X, R(Y)], R_{-}=R-1$, и то, что $\nabla I_{k}(L)$ есть инвариантная функция, а потому $\forall X \in \mathfrak{g}$ справедливо равенство $\left\langle R_{+}^{*}(L),\left[\left.\nabla I_{k}(L)\right|_{L=R_{+}^{*}(L)}, X\right]\right\rangle=0$, нетрудно показать, что первое слагаемое в правой части выражения (16) может быть преобразовано к виду

$$
2\left\langle R_{+}^{*}(L),\left[\left.R_{-} \nabla I_{k}(L)\right|_{L=R_{+}^{*}(L)}, R_{-}\left(\left.\nabla I_{l}(L)\right|_{L=R_{-}^{*}(L)}\right)\right]\right\rangle .
$$

Аналогично, получим

$$
\begin{aligned}
&\left\langle R_{-}^{*}(L),[\right.\left.\left.R_{+}\left(\left.\nabla I_{k}(L)\right|_{L=R_{+}^{*}(L)}\right), R_{-}\left(\left.\nabla I_{l}(L)\right|_{L=R_{-}^{*}(L)}\right)\right]_{R}\right\rangle= \\
&=- 2\left\langle R_{-}^{*}(L),\left[R_{+}\left(\left.\nabla I_{k}(L)\right|_{L=R_{+}^{*}(L)}\right), R_{+}\left(\left.\nabla I_{l}(L)\right|_{L=R_{-}^{*}(L)}\right)\right]\right\rangle+ \\
& \quad+\left\langle R_{-}^{*}(L),\left[R_{+}\left(\left.\nabla I_{k}(L)\right|_{L=R_{+}^{*}(L)}\right), R_{+}\left(\left.\nabla I_{l}(L)\right|_{L=R_{-}^{*}(L)}\right)\right]_{R}\right\rangle .
\end{aligned}
$$

В итоге имеет место равенство

$$
\begin{aligned}
2\left\{I_{k}^{R_{+}}\right. & \left.(L), I_{l}^{R_{-}}(L)\right\}_{R}=2\left(\left\langle R_{+}^{*}(L),\left[\left.R_{-} \nabla I_{k}(L)\right|_{L=R_{+}^{*}(L)}, R_{-}\left(\left.\nabla I_{l}(L)\right|_{L=R_{-}^{*}(L)}\right)\right]\right\rangle+\right. \\
& \left.+\left\langle R_{-}^{*}(L),\left[R_{+}\left(\left.\nabla I_{k}(L)\right|_{L=R_{+}^{*}(L)}\right), R_{+}\left(\left.\nabla I_{l}(L)\right|_{L=R_{-}^{*}(L)}\right)\right]\right\rangle\right)+ \\
& +\left\langle R_{+}^{*}(L),\left[R_{-}\left(\left.\nabla I_{k}(L)\right|_{L=R_{+}^{*}(L)}\right), R_{-}\left(\left.\nabla I_{l}(L)\right|_{L=R_{-}^{*}(L)}\right)\right]_{R}\right\rangle- \\
& -\left\langle R_{-}^{*}(L),\left[R_{+}\left(\left.\nabla I_{k}(L)\right|_{L=R_{+}^{*}(L)}\right), R_{+}\left(\left.\nabla I_{l}(L)\right|_{L=R_{-}^{*}(L)}\right)\right]_{R}\right\rangle
\end{aligned}
$$

Используя равенства (3в) и $\left[R_{ \pm}, R\right]=0$, нетрудно показать, что

$$
R_{-}\left(\left[R_{+}(X), R_{+}(Y)\right]_{R}\right)=R_{+}\left(\left[R_{-}(X), R_{-}(Y)\right]_{R}\right) .
$$


С учетом этого равенства легко показать, что два последних слагаемых в выражении (17) для $\left\{I_{k}^{R_{+}}(L), I_{l}^{R_{-}}(L)\right\}_{R}$ взаимно сокращаются, а первые два упрощаются и принимают вид

$$
\left\{I_{k}^{R_{+}}(L), I_{l}^{R_{-}}(L)\right\}_{R}=2\left\langle R_{+}^{*}(L),\left[\left.R_{-} \nabla I_{k}(L)\right|_{L=R_{+}^{*}(L)}, R_{-}\left(\left.\nabla I_{l}(L)\right|_{L=R_{-}^{*}(L)}\right)\right]\right\rangle .
$$

Применяя равенство (3б), окончательно получим для $\left\{I_{k}^{R_{+}}(L), I_{l}^{R_{-}}(L)\right\}_{R}$ следующее простое выражение:

$$
\left\{I_{k}^{R_{+}}(L), I_{l}^{R_{-}}(L)\right\}_{R}=2\left\langle L, R_{+} R_{-}\left(\left[\left.\nabla I_{k}(L)\right|_{L=R_{+}^{*}(L)},\left.\nabla I_{l}(L)\right|_{L=R_{-}^{*}(L)}\right]_{R}\right)\right\rangle .
$$

Выполняя очевидные преобразования, можно переписать выражение (15) в следующем виде:

$$
\left\{I_{k}^{R_{ \pm}}(L), I_{l}^{R_{ \pm}}(L)\right\}_{R}=2\left\langle L,\left(R_{ \pm}^{2} \mp 2 R_{ \pm}\right)\left(\left[\left.\nabla I_{k}(L)\right|_{L=R_{ \pm}^{*}(L)},\left.\nabla I_{l}(L)\right|_{L=R_{ \pm}^{*}(L)}\right]_{R}\right)\right\rangle .
$$

Пусть теперь $R$ имеет тип KАС, т.е. $R=P_{+}-P_{-}$. Тогда $R_{+} R_{-}=0$ и, следовательно, $\left\{I_{k}^{R_{+}}(L), I_{l}^{R_{-}}(L)\right\}_{R}=0$. Более того, в этом случае $R_{ \pm}^{2}= \pm 2 R_{ \pm}$, а потому $\left\{I_{k}^{R_{ \pm}}(L), I_{l}^{R_{ \pm}}(L)\right\}_{R}=0$. Это завершает доказательство теоремы в случае, когда $R$ имеет тип $\mathrm{KAC}$.

Пусть теперь оператор $R$ получен из треугольного разложения, т.е. $R=P_{+}+R_{0}-$ $P_{-}$. В этом случае нетрудно найти, что $R_{+} R_{-}=\left(R_{0}\right)_{+}\left(R_{0}\right)_{-}$, где $\left(R_{0}\right)_{+}=R_{0}+P_{0}$, $\left(R_{0}\right)_{-}=R_{0}-P_{0}$. В этом случае с помощью простых вычислений получим ответ: $R_{ \pm}^{2}= \pm 2 R_{ \pm}+\left(R_{0}\right)_{ \pm}^{2} \mp 2\left(R_{0}\right)_{ \pm}$. Кроме того, прямыми вычислениями получим, что

$$
\begin{gathered}
{[X, Y]_{R}=2\left(\left[X_{+}, Y_{+}\right]-\left[X_{-}, Y_{-}\right]\right)+\left[\left(R_{0}+1\right)\left(X_{0}\right), Y_{+}\right]+\left[X_{+},\left(R_{0}+1\right)\left(Y_{0}\right)\right]+} \\
+\left[\left(R_{0}-1\right)\left(X_{0}\right), Y_{-}\right]+\left[X_{-},\left(R_{0}-1\right) Y_{0}\right]+\left[X_{0}, Y_{0}\right]_{R_{0}} .
\end{gathered}
$$

Учитывая все вышесказанное и то, что $\mathfrak{g}_{ \pm}$представляют собой $\mathfrak{g}_{0}$-модули и что $\left(R_{0}\right)_{ \pm}$по определению действует нетривиально только на $\mathfrak{g}_{0}$, получим, что равенства (18), (19) принимают следующий вид:

$$
\begin{aligned}
& \left\{I_{k}^{R_{+}}(L), I_{l}^{R_{-}}(L)\right\}_{R}= \\
& \quad=4\left\langle L,\left(R_{0}\right)_{+}\left(R_{0}\right)_{-}\left(\left[P_{0}\left(\left.\nabla I_{k}(L)\right|_{L=R_{+}^{*}(L)}\right), P_{0}\left(\left.\nabla I_{l}(L)\right|_{L=R_{-}^{*}(L)}\right)\right]_{R_{0}}\right)\right\rangle, \\
& \left\{I_{k}^{R_{ \pm}}(L), I_{l}^{R_{ \pm}}(L)\right\}_{R}= \\
& \quad=2\left\langle L,\left(\left(R_{0}\right)_{ \pm}^{2} \mp 2\left(R_{0}\right)_{ \pm}\right)\left(\left[P_{0}\left(\left.\nabla I_{k}(L)\right|_{L=R_{ \pm}^{*}(L)}\right), P_{0}\left(\left.\nabla I_{l}(L)\right|_{L=R_{ \pm}^{*}(L)}\right)\right]_{R_{0}}\right)\right\rangle
\end{aligned}
$$

где $P_{0}$ - проекционный оператор на подалгебру $\mathfrak{g}_{0}$.

В результате получим, что эти выражения тождественно обращаются в нуль в следующих случаях.

1. Если $\mathfrak{g}_{0}$ - абелева подалгебра.

2 . Если $\left(R_{0}\right)_{+}\left(R_{0}\right)_{-}=0$ и $\left(\left(R_{0}\right)_{ \pm}^{2} \mp 2\left(R_{0}\right)_{ \pm}\right)=0$, т.е. если оператор $R_{0}$ получен из разложения $\mathfrak{g}_{0}$ типа $\mathrm{KAC}$. Но в этом случае оператор $R$ также получается из 
разложения типа $\mathrm{KAC}$ алгебры $\mathfrak{g}$ и может быть записан в виде $R=\left(P_{+}+\left(P_{0}\right)_{+}\right)-$ $\left(P_{-}+\left(P_{0}\right)_{-}\right)$, где $\mathfrak{g}_{0}=\left(\mathfrak{g}_{0}\right)_{+}+\left(\mathfrak{g}_{0}\right)_{-}$и $\left(P_{0}\right)_{ \pm}-$проекционные операторы на соответствующие алгебры Ли $\left(\mathfrak{g}_{0}\right)_{ \pm}$.

3 . Оператор $R_{0}$ получается из треугольного разложения $\mathfrak{g}_{0}: \mathfrak{g}_{0}=\left(\mathfrak{g}_{0}\right)_{+}+\left(\mathfrak{g}_{0}\right)_{0}+$ $\left(\mathfrak{g}_{0}\right)_{-}$, в котором подалгебра $\left(\mathfrak{g}_{0}\right)_{0}$ абелева. В этом случае оператор $R$ получается из треугольного разложения $\mathfrak{g}$ с абелевой подалгеброй $\mathfrak{g}_{0}^{\prime} \equiv\left(\mathfrak{g}_{0}\right)_{0}$ и может быть записан в виде $R=P_{+}+\left(P_{0}\right)_{+}+\left(R_{0}\right)_{0}-P_{-}-\left(P_{0}\right)_{-}$.

Теорема доказана.

\section{4. СВЯЗЬ С ИНТЕГРИРУЕМЫМИ ГАМИЛЬТОНОВЫМИ СИСТЕМАМИ}

Теорема 1 описывает набор взаимно коммутирующих функций на $\mathfrak{g}^{*}$ относительно скобок $\{\cdot, \cdot\}$, который можно использовать в качестве алгебры интегралов движения некоторой гамильтоновой системы на $\mathfrak{g}^{*}$. В качестве функции Гамильтона можно выбрать одну из функций $I_{k}^{R_{ \pm}}(L)$ или их линейную комбинацию. Рассмотрим следующее гамильтоново уравнение:

$$
\frac{d L_{i}}{d t_{k}^{ \pm}}=\left\{L_{i}, I_{k}^{R_{ \pm}}(L)\right\}
$$

Покажем, что гамильтоновы уравнения (20) могут быть записаны в форме пары Лакса. Имеет место следующее предложение.

ПрЕДЛОЖЕНИЕ 2. Гамильтоновы уравнения движения (20) могут быть записаны в форме Эйлера-Арнольда (обобщенной лаксовой форме)

$$
\frac{d L}{d t_{k}^{ \pm}}=\operatorname{ad}_{M}^{*} L
$$

где $M \equiv \nabla I_{k}^{R_{ \pm}}(L)=R_{ \pm}\left(\left.\nabla I_{k}(L)\right|_{L=R_{ \pm}^{*}(L)}\right)$.

ДокАЗАтЕльство. Непосредственным преобразованием получим

$$
\frac{d L_{i}}{d t_{k}^{ \pm}}=\left\{L_{i}, I_{k}^{R_{ \pm}}(L)\right\}=\left\langle L,\left[\nabla I_{k}^{R_{ \pm}}(L), X_{i}\right]\right\rangle=\left\langle\operatorname{ad}_{\nabla I_{k}^{R_{ \pm}}(L)}^{*} L, X_{i}\right\rangle .
$$

Умножая обе части на $X_{i}^{*}$ и суммируя по всем $i \in\left\{1, \ldots, \operatorname{dim} \mathfrak{g}^{*}\right\}$, приходим к утверждению предложения.

ЗАмечАниЕ 7 . В случае стандартной $R$-матричной интегрируемости имеют место гамильтоновы уравнения движения для гамильтониана $I_{k}(L)$ относительно $R$-скобки:

$$
\frac{d L_{i}}{d \tau_{k}}=\left\{L_{i}, I_{k}(L)\right\}_{R} .
$$

Они также могут быть записаны в обобщенном лаксовом виде, но с другим $M$-оператором [1]:

$$
\frac{d L}{d \tau_{k}}=\operatorname{ad}_{M}^{*} L, \quad M=R\left(\nabla I_{k}(L)\right)
$$


Чтобы получить полностъю интегрируемые гамильтоновы системы в случае конечномерных алгебр Ли $\mathfrak{g}$ в рамках нашего подхода, необходимо потребовать, чтобы число построенных функционально независимых интегралов было бы равно половинной размерности фазовых пространств, которые представляют собой симплектические листы коприсоединенных орбит группы $G$ в $\mathfrak{g}^{*}$. С другой стороны, нетрудно видеть, что число функционально независимых интегралов, полученных в рамках нашего подхода, равно 2 ind $\mathfrak{g}$. Поэтому если только $\mathfrak{g}$ - полупростая (редуктивная) конечномерная алгебра Ли ранга $r(r=$ ind $\mathfrak{g})$, то наша конструкция задает полностью интегрируемые системы на $\mathfrak{g}$ в случае достаточно малых $r$ или в случае достаточно вырожденных коприсоединенных орбит в $\mathfrak{g}^{*}$.

Другое возможное приложение к теории конечномерных интегрируемых систем состоит в рассмотрении гамильтоновых систем на подпространствах $\left(\mathfrak{g}_{R_{+}}^{*},\{\cdot, \cdot\}\right)$ или $\left(\mathfrak{g}_{R_{-}}^{*},\{\cdot, \cdot\}\right)$ с абелевой алгеброй Ли интегралов движения, порожденной только одним из наборов функций $I_{k}^{R_{+}}(L)$ или $I_{k}^{R_{-}}(L)$.

Наконец, еще одно возможное применение наших результатов к теории конечномерных интегрируемых систем состоит в рассмотрении гамильтоновых систем на $\mathfrak{g}^{*}$ со скобкой Пуассона $\{\cdot, \cdot\}_{R}$ в случае, когда $I_{k}^{R_{+}}(L)$ и $I_{k}^{R_{-}}(L)$ порождают абелеву подалгебру также и относительно скобки $\{\cdot, \cdot\}_{R}$. В случае, когда $R$ не является оператором типа KAC, это приводит к гамильтоновым системам, которые не сводятся к обсуждавшимся выше.

Тем не менее представляется вероятным, что наиболее интересные и перспективные применения наших результатов лежат в области теорий бесконечномерных интегрируемых систем, когда алгебра $\mathfrak{g}$ бесконечномерна. В этом случае наши интегралы и их гамильтоновы потоки можно использовать для построения новых солитонных решений в рамках техники, сходной с техникой работы [3] (см. также [4]). Автор предполагает вернуться к этой задаче [6].

Благодарности. Автор признателен П. Голоду и Д. Лейкину за полезные обсуждения. Работа частично поддержана целевой программой Отделения физики и астрономии Национальной академии наук Украины, а также DFFD (грант № $\mathrm{F} / 16-457-2007)$.

\section{Список литературы}

[1] М. А. Семёнов-Тян-Шанский, Функи. анализ и его прил., 17:4 (1983), 17-33.

[2] А. Г. Рейман, М. А. Семенов-Тян-Шанский, "Теоретико-групповые методы в теории конечномерных интегрируемых систем", Интегрируемые системы. Неголономные динамические системы, Серия современные проблемы математики. Фундаментальные направления, 16, ред. В. И. Арнольд, С. П. Новиков, ВИНИТИ, М., 1987, 119-193.

[3] A. Newell, Solitons in Mathematics and Physics, CBMS-NSF Regional Conference Series in Applied Mathematics, 48, SIAM, Philadelphia, PA, 1985.

[4] Т. В. Скрыпник, ТМФ, 142:2 (2005), 329-345.

[5] F. Guil, M. Manas, Lett. Math. Phys., 19 (1990), 89-95.

[6] T. Skrypnyk, SIGMA, 04 (2008), 011.

[7] A. Belavin, V. Drienfield, preprint ITF 1982-18, ITF, Chernogolovka.

[8] А. А. Белавин, В. Г. Дринфельд, Функи. анализ и его прил., 16:3 (1982), 159-180. 